\title{
Papel da Histerossonografia no Estudo da Cavidade Uterina em Pacientes com Sangramento Uterino Anormal
}

\author{
Role of Sonohysterography in the Evaluation of the Uterine Cavity in Patients \\ with Abnormal Uterine Bleeding
}

Angélica Lemos Debs Diniz, Elmar Gonzaga Gonçalves

\begin{abstract}
RESUMO
Objetivo: determinar o papel da histerossonografia na avaliação das anormalidades da cavidade uterina, em pacientes com sangramento uterino anormal, selecionadas previamente pela ultra-sonografia transvaginal.

Métodos: foram selecionadas 48 pacientes na menacme e pós-menopausa, portadoras de sangramento uterino anormal e anormalidades da cavidade uterina, diagnosticadas inicialmente pela ultra-sonografia transvaginal. Todas as pacientes se submeteram à histerossonografia e, posteriormente, aos métodos "padrão ouro", a histeroscopia e/ou histerectomia. As histerossonografias foram avaliadas por dois diferentes médicos e os diagnósticos confrontados.

Resultados: a histerossonografia mostrou ter alta sensibilidade e especificidade no diagnóstico das patologias benignas da cavidade uterina. Na presença de pólipo a sensibilidade e a especificidade do método foram, respectivamente, de 100 e 97\%, seguido do mioma submucoso cuja sensibilidade e especificidade foram, respectivamente, de $83 e$ 100\%. Já na hiperplasia endometrial e no endométrio normal a sensibilidade e especificidade foram de 100\%. Diagnosticamos 33 casos de pólipos, 13 casos de miomas submucosos, quatro casos de hiperplasia endometrial e três casos normais na análise dos métodos "padrão ouro". Houve alta correlação entre os diagnósticos dados pelos dois examinadores.

Conclusões: a histerossonografia mostrou ser um exame reprodutivel, com alta sensibilidade e especificidade no diagnóstico das patologias benignas da cavidade uterina em pacientes com sangramento uterino anormal.
\end{abstract}

PALAVRAS-CHAVE: Ultra-sonografia transvaginal. Hemorragia uterina. Histerossonografia.

\section{Introdução}

As enfermidades hemorrágicas ginecológicas continuam sendo uma das maiores causas de procura por consultório ginecológico e são responsáveis por $20 \%$ das consultas novas ${ }^{1}$.

Atualmente, com o início precoce da menarca, o uso de métodos contraceptivos para

Universidade Federal de Uberlândia

Correspondência:

Angélica Lemos Debs Diniz

Al. João César de Souza, 110 - Morada da Colina

38411-154 - Uberlândia - MG

e-mail: Angelyca@net.em.com.br limitar as gestações e um número cada vez maior de mulheres vivendo longos períodos após a menopausa, houve um acréscimo na média de ciclos menstruais para cerca de $400^{2}$. Assim, as alterações menstruais passaram a ser queixas cada vez mais freqüentes tanto para os ginecologistas, quanto para os médicos das áreas de diagnóstico por imagem. Em casos de pacientes com sangramento uterino anormal (SUA), a avaliação do útero, endométrio e ovários por um método de imagem é fundamental para a identificação das anormalidades orgânicas.

A investigação da cavidade uterina vem apresentando rápida evolução, desde o apareci- 
mento da ultra-sonografia transvaginal ${ }^{3}$. Nenhuma outra técnica apresentou avanços tão excepcionais e extensos quanto a ultra-sonografia na área ginecológica. O campo de ação da ultrasonografia para a avaliação endometrial parecia já estar esgotado, quando foi surpreendido pela descrição de uma nova técnica denominada histerossonografia (HSF). Nannini et $a{ }^{4}{ }^{4}$, foram os primeiros a descrever este procedimento em 1981, ao observarem que a injeção de solução salina intra-útero favorecia a visibilização da cavidade uterina. Três anos após, Richman et al. ${ }^{5}$ avaliaram 35 pacientes inférteis, realizando a histerossalpingo-sonografia, com o objetivo principal de estudar a patência tubária. O resultado deste estudo foi que o método em questão, além de ser sensivel na detecção de obstruções tubárias, também foi sensivel na avaliação da cavidade uterina. Randolph et al. ${ }^{6}$ em 1986 empregaram a injeção de solução salina intra-útero em conjunto com a ultra-sonografia abdominal num grupo de pacientes inférteis, e conseguiram diagnosticar corretamente 53 das 54 anormalidades da cavidade uterina, aumentando, assim, a credibilidade do método. Todos estes autores se aproveitaram do fato de a cavidade uterina ter fácil acesso à introdução de cateteres através do colo. A partir destes primeiros estudos foi possivel observar uma tendência à superioridade das imagens obtidas pela histerossonografia em relação à ultrasonografia convencional, levando, assim, a um maior detalhamento em termos de localização, textura e biometria das alterações endometriais.

Baseado nos estudos realizados até o momento, acredita-se que a HSF abrirá novas perspectivas no que diz respeito ao diagnóstico das doenças endometriais, com conseqüente redução do número de procedimentos invasivos, favorecendo a condução segura e menos onerosa dos casos de SUA.

O objetivo deste estudo é determinar o valor da histerossonografia na avaliação das anormalidades da cavidade uterina em pacientes com sangramento uterino anormal.

\section{Pacientes e Métodos}

Trata-se de estudo clínico transversal, realizado no Setor de Ultra-Sonografia do Hospital de Clínicas da Universidade Federal de Uberlândia, em conjunto com o CDE - Centro de Diagnósticos Ecográficos, que analisou o papel da histerossonografia em um grupo de 48 pacientes com SUA, portadoras de anormalidades da cavidade uterina. Os diagnósticos das histerossonografias foram reali- zados por dois diferentes examinadores e comparados entre si, além de serem comparados com os resultados obtidos pelos métodos "padrão ouro".

Os critérios clínicos básicos para a inclusão no estudo foram: a) ausência de sinais clínicos de infecção vaginal ou doença inflamatória pélvica aguda; b) pacientes na menacme com sangramento vaginal anormal, incluindo somente os seguintes desvios menstruais para mais: menorragia, metrorragia, polimenorréia, hipermenorréia e sinusorragia; c) pacientes na pós-menopausa, com sangramento vaginal, independentemente da duração e/ou volume. A menopausa foi definida como amenorréia de, no minimo, 6 meses. As pacientes poderiam ou não estar sob uso de qualquer esquema de terapia de reposição hormonal.

A avaliação ultra-sonográfica foi realizada com o uso de equipamentos Aloka 2000 Multi View, com transdutor transvaginal eletrônico, convexo, na freqüência de $5 \mathrm{MHz}$ e Siemens Sonoline, versa Pro, com transdutor transvaginal eletrônico, convexo, na freqüência de $6 \mathrm{MHz}$.

Os critérios ultra-sonográficos utilizados para definir as anormalidades da cavidade uterina foram: a) endométrio com espessura incompatível com a fase do ciclo na menacme; b) endométrio irregular; c) massas heterogêneas na cavidade uterina; d) miomas submucosos, levando à compressão extrinseca do endométrio; e) alterações endometriais ecograficamente inespecíficas; f) endométrio com espessura igual ou superior a 10 $\mathrm{mm}$ em pacientes na pós-menopausa sob terapia de reposição hormonal; g) endométrio com espessura igual ou superior a $5 \mathrm{~mm}$ em pacientes na pós-menopausa, sem terapia de reposição hormonal.

A histerossonografia foi realizada com a paciente em posição de litotomia, introdução de um espéculo vaginal e anti-sepsia com solução de Povidine tópico. Em seguida, foi inserida sonda de Foley, número oito, ou cateter de Freedman ou sonda de histerossonografia (ZUI - $2 \mathrm{~mm}$ ) no canal endocervical, até ocorrer a transposição do orificio interno. O balão da sonda era então insuflado (exceto no cateter de Freedman) e o espéculo vaginal retirado, com posterior introdução do transdutor transvaginal para o início do estudo ultra-sonográfico da cavidade uterina. Durante o exame, injetou-se soro fisiológico $0,9 \%$, lentamente, até obter-se a distensão satisfatória da cavidade uterina. Todas as pacientes foram informadas sobre o caráter científico da histerossonografia. As HSF foram fotografadas e gravadas para análise dinâmica. O tempo máximo permitido entre a realização da histerossonografia e a histeroscopia e/ou histerectomia foi de três meses.

Solicitou-se às pacientes que informassem 
ao médico caso ocorresse, após o procedimento, febre $\left(\mathrm{T}>38^{\circ}\right)$, leucorréia amarelada, sangramento vaginal importante ou dor pélvica persistente.

Dois ultra-sonografistas experientes avaliaram primeiramente as imagens gravadas da ultra-sonografia transvaginal, formulando uma hipótese diagnóstica para as alterações endometriais encontradas. A seguir, os mesmos avaliaram as imagens gravadas da histerossonografia e definiram nova hipótese diagnóstica. Os examinadores não tiveram acesso prévio aos resultados da histeroscopia ou do anatomopatológico da peça cirúrgica. Os diagnósticos feitos pela HSG foram confrontados com os resultados das histeroscopias e anatomopatológicos. Os métodos definidos como "padrão ouro" para as alterações endometriais foram a histeroscopia em conjunto com o exame anatomopatológico das peças operatórias.

Os dados deste estudo foram apresentados de forma descritiva, utilizando-se porcentagem, média aritmética e desvio padrão, conforme apropriado. Foram determinadas a sensibilidade, a especificidade e os valores preditivos positivo e negativo da histerossonografia, por patologias encontradas. Para cada proporção foi estabelecido o intervalo de confiança (IC) de 95\%.

Com o objetivo de verificarmos se houve concordância diagnóstica interobservador das histerossonografias e destas com os métodos "padrão ouro", aplicou-se o índice Kappa (IK) ${ }^{7}$.

\section{Resultados}

A média aritmética da idade foi de $47 \pm 12$ anos (30 a 79 anos). Diante das histerossonografias analisadas pelo examinador I, foram feitos os seguintes diagnósticos: pólipo em 37 casos $(72,5 \%)$, mioma em 7 casos $(13,7 \%)$, hiperplasia endometrial em 4 casos $(7,8 \%)$ e normal em 3 casos $(5,9 \%)$. Já diante das HSF analisadas pelo examinador II, os diagnósticos foram: pólipo em 34 casos $(66,6 \%)$, mioma em 10 casos $(19,6 \%)$, hiperplasia em 4 casos $(7,8 \%)$ e normal em 3 casos (5,9\%) (Tabela 1$)$.

Tabela 1 - Diagnósticos das histerossonografias analisadas pelos examinadores I e I| nas 48 pacientes com sangramento uterino anormal.

\begin{tabular}{lcc}
\hline Alterações & $\begin{array}{c}\text { Examinador I } \\
\mathbf{n}\end{array}$ & $\begin{array}{c}\text { Examinador II } \\
\mathbf{n}\end{array}$ \\
\hline Pólipo & 37 & 34 \\
Mioma & 7 & 10 \\
Hiperplasia & 4 & 4 \\
Normal & 3 & 3 \\
Total & 51 & 51 \\
\hline
\end{tabular}

O nível de concordância interobservador da HSF está expresso abaixo pelo índice Kappa:

1- pólipo: $\mathrm{IK}=0,90$;

2- mioma: $\mathrm{IK}=0,89$;

3- hiperplasia: $\mathrm{IK}=1,0$;

4- normal: $\mathrm{IK}=1,0$.

A determinação da sensibilidade, especificidade, valor preditivo positivo e valor preditivo negativo da histerossonografia foi feita somente para os resultados do examinador II, devido ao alto nivel de concordância interobservador encontrado pelo método.

A sensibilidade da HSF na detecção de pólipo foi de 100\% (IC: 0,96-1,00), com especificidade de 93\% (IC: 0,93-1,07), valor preditivo positivo de $97 \%$ (IC: $0,92-1,02$ ) e valor preditivo negativo de $100 \%$ (IC: 0,96-1,00) (Tabela 2). Houve um caso diagnosticado como pólipo pela histerossonografia que mostrou ser adenomioma pelo estudo anatomopatológico (Figura 1).

Tabela 2 - Diagnósticos de pólipos feitos por meio da análise das 48 histerossonografias (HSF) realizadas no grupo de pacientes com sangramento uterino anormal.

\begin{tabular}{lcc}
\hline & $\begin{array}{c}\text { "Padrão ouro" } \\
\text { com pólipo }\end{array}$ & $\begin{array}{c}\text { "Padrão ouro" } \\
\text { sem pólipo }\end{array}$ \\
\hline HSF com pólipo & 33 & 1 \\
HSF sem pólipo & 0 & 14 \\
\hline
\end{tabular}

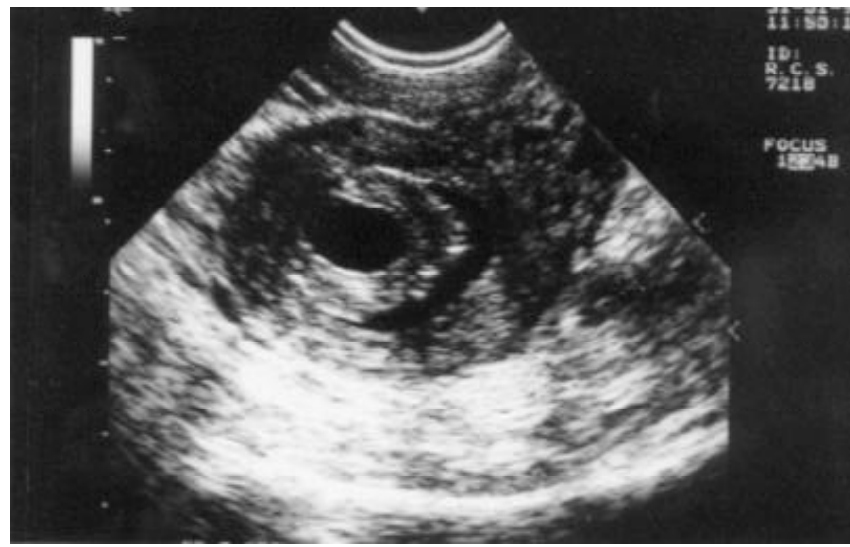

Figura 1 - Adenomioma diagnosticado como pólipo pela histerossonografia. Presença de grande imagem nodular ecogênica, com centro anecóico, fazendo projeção para a cavidade uterina.

No diagnóstico de mioma submucoso a sensibilidade foi de $83 \%$ (IC: $0,64-0,88$ ), com especificidade de $100 \%$ (IC: 0,96-1,00), valor preditivo positivo de 100\% (IC: 0,96-1,00) e valor preditivo negativo de 94\% (IC: 0,77-0,96) (Tabela 3). Em dois casos não se visibilizou a concomitância do mioma 
submucoso com o pólipo endometrial, falhando, assim, no diagnóstico completo das patologias da cavidade uterina (Figuras 2 e 3 ). No terceiro caso, o diagnóstico dado pela HSF foi de pólipo necrosado, sendo que o estudo anatomopatológico obtido após a HTA diagnosticou um adenomioma. Este caso foi incluído no estudo como mioma submucoso pelo fato de a tumoração consistir mais de componentes miomatosos que glandulares.

Tabela 3 - Diagnósticos de miomas submucosos feitos por meio da análise das 48 histerossonografias (HSF) realizadas no grupo de pacientes com sangramento uterino anormal.

\begin{tabular}{lcc}
\hline & $\begin{array}{c}\text { "Padrão ouro" } \\
\text { com mioma }\end{array}$ & $\begin{array}{c}\text { "Padrão ouro" } \\
\text { sem mioma }\end{array}$ \\
\hline HSF com mioma & 10 & 0 \\
HSF sem mioma & 3 & 35 \\
\hline
\end{tabular}

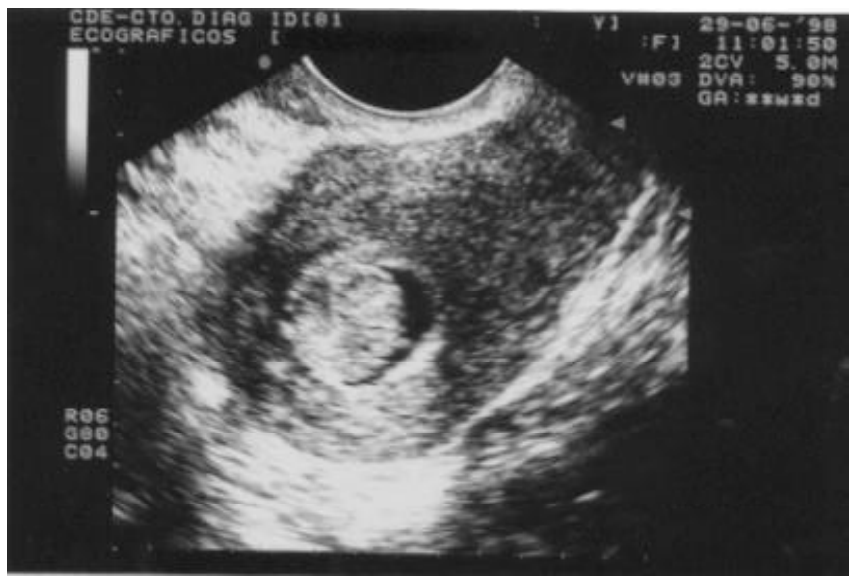

Figura 2 - Histerossonografia mostrando apenas uma imagem nodular e hiperecogênica, localizada no fundo da cavidade uterina, compatível com pólipo. Neste caso havia pequeno mioma submucoso que não foi identificado.

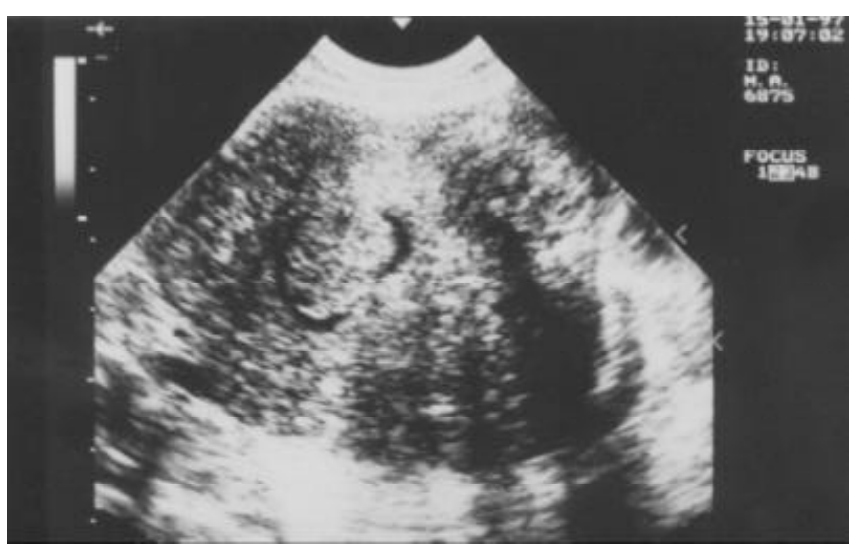

Figura 3 - Nota-se mioma intramural na parede posterior do útero e imagem ecogênica, fazendo protusão na parede anterior da cavidade, compativel com pólipo. Neste caso havia um pequeno mioma submucoso que não foi diagnosticado.
$\mathrm{Na}$ análise da presença de hiperplasia endometrial e cavidade normal, a correlação da histerossonografia com o método "padrão ouro" foi de $100 \%$, a sensibilidade, a especificidade e os valores preditivos positivo e negativo foram de $100 \%$ (IC: 0,96-1,00).

Obtivemos, portanto, um diagnóstico falsopositivo para pólipo e três falso-negativos para mioma submucoso.

A Tabela 4 mostra e compara a sensibilidade, a especificidade, e os valores preditivos positivo e negativo da histerossonografia, diante de cada patologia da cavidade uterina.

Tabela 4 - Análise da sensibilidade, especificidade e valores preditivos positivo e negativo da histerossonografia (HSF).

\begin{tabular}{lccc}
\hline $\begin{array}{l}\text { Anormalidades } \\
(\mathrm{HSF} / \mathrm{PO})\left(\mathrm{n}^{\circ}\right)\end{array}$ & $\begin{array}{c}\text { Pólipo } \\
\mathbf{3 4 / 3 3}\end{array}$ & $\begin{array}{c}\text { Mioma } \\
10 / 13\end{array}$ & $\begin{array}{c}\text { Hiperplasia } \\
\mathbf{4} / \mathbf{4}\end{array}$ \\
\hline Sensibilidade (\%) & 100 & 83 & 100 \\
Especificidade (\%) & 93 & 100 & 100 \\
VPP (\%) & 97 & 100 & 100 \\
VPN (\%) & 100 & 94 & 100 \\
\hline
\end{tabular}

VPP: Valor preditivo positivo; VPN: valor preditivo negativo PO: "Padrão ouro"

O índice Kappa também foi aplicado na análise da concordância entre os diagnósticos da HSF e dos métodos "padrão ouro", com os seguintes resultados:

1- pólipo: $\mathrm{IK}=0,95$

2- mioma: $\mathrm{IK}=0,90$;

3- hiperplasia: $\mathrm{IK}=1,0$;

4- normal: $\mathrm{IK}=1,0$.

A histeroscopia e o estudo anatomopatológico do útero após a histerectomia mostraram as seguintes alterações: pólipos em 33 casos, miomas submucosos em 13 casos, hiperplasia endometrial em quatro casos, e normal em três casos, totalizando 53 diagnósticos.

Foram realizadas seis histerectomias abdominais e 45 histeroscopias. Em 3 casos, a histeroscopia foi realizada previamente à histerectomia abdominal.

\section{Discussão}

Desde a publicação inicial feita por Nannini et al. em $1981^{4}$, um número não muito grande de trabalhos foi publicado sobre o papel da histerossonografia em mulheres na menacme e na pós- 
menopausa, com quadros de sangramento uterino anormal.

Neste estudo, a amostra selecionada foi composta exclusivamente por pacientes portadoras de SUA, por ser esta uma das queixas mais freqüentes nos consultórios ginecológicos ${ }^{1}$. Sabese que a ultra-sonografia transvaginal é um método fundamental no rastreamento das patologias uterinas e endometriais de pacientes com alterações menstruais, independentemente da fase em que se encontram (menacme, perimenopausa ou pós-menopausa), além de ser reprodutive1 ${ }^{8-10}$. Portanto, optou-se por usar a ultra-sonografia transvaginal na seleção das anormalidades da cavidade uterina em pacientes com sangramento uterino anormal. Não há dúvidas de que discretas patologias endometriais foram subdiagnosticadas pela ultra-sonografia transvaginal e excluídas deste estudo, mas a seleção das pacientes com a ultrasonografia transvaginal foi base fundamental para que pudéssemos obter o consentimento das pacientes na realização da histerossonografia. Estamos atentos ao fato de que esta seleção das pacientes traz algumas limitações no que diz respeito à interpretação dos resultados deste estudo.

Quanto à determinação do método "padrão ouro", não há dúvidas de que o estudo anatomopatológico da peça cirúrgica é o ideal para a confirmação da presença de patologias uterinas e endometriais. Entretanto, a indicação de histerectomia foi restrita a poucos casos, sendo associada a histeroscopia como método "padrão ouro", por ter alta sensibilidade e especificidade na detecção de anormalidades da cavidade uterina ${ }^{11}$.

Nesta amostra, 24\% das pacientes com SUA encontravam-se na pós-menopausa, mas não se detectou nenhum caso de patologia endometrial maligna, o que era esperado, tendo em vista o pequeno número de pacientes avaliadas.

Dentro dos quatro diagnósticos feitos pela HSF, nesta série de 48 pacientes com sangramento uterino anormal, o pólipo e o mioma juntos representaram $86,3 \%$ dos casos e a hiperplasia endometrial e os casos normais somaram 13,7\%. A histerossonografia mostrou ser importante na exclusão de anormalidade em três casos, cuja ultra-sonografia transvaginal diagnosticou erroneamente patologias da cavidade uterina. A sensibilidade e especificidade da HSG foram respectivamente 100 e 93\% na detecção de pólipos, o que está de acordo com os achados da literatura ${ }^{12-14}$. A histerossonografia, além de detectar todos os pólipos, possibilitou a fiel localização da base dos mesmos, facilitando a realização de tratamentos cirúrgicos, além de reduzir os diagnósticos falsonegativos das biópsias orientadas ${ }^{14}$. Obtiveram-se neste estudo dois casos de pequenos pólipos, des- critos na literatura como prega endometrial ou "wrinkles" ${ }^{14}$, sendo que estes pólipos dificilmente são diagnosticados pela ultra-sonografia transvaginal. As pregas endometriais, apesar de pequenas, têm grande importância no que diz respeito à sintomatologia, podendo levar a quadros de SUA, que só melhoram após a ressecção cirúrgica desta área.

Já no que diz respeito à sensibilidade da HSF no diagnóstico de mioma submucoso, o valor encontrado neste estudo foi inferior ao descrito na literatura, mas a especificidade foi alta e está de acordo com a maioria dos autores ${ }^{15-17}$. A baixa sensibilidade obtida neste estudo pode ser explicada pelo fato de haver no caso 1 uma imagem inespecífica, tanto na histerossonografia, quanto na histeroscopia, e de esta representar uma patologia pouco freqüente que é o adenomioma, tumor composto por tecido miomatoso e glandular. Sabese que qualquer estrutura que faz proeminência para a cavidade uterina pode ser chamada de pólipo, e a histeroscopia também poderá ser limitada na definição da presença do verdadeiro pólipo, indicando que nem toda estrutura com aparência polipóide corresponderá ao achado histopatológico de pólipo endometrial ${ }^{17}$. No segundo e terceiro casos em que houve falha no diagnóstico de mioma submucoso, eles eram muito pequenos e quase não levavam ao abaulamento da cavidade uterina, não sendo, assim, valorizados pelo examinador. Fukuda et al. ${ }^{16}$ avaliaram 36 pacientes com hipermenorréia, dismenorréia e/ou anemia e relataram uma sensibilidade de $100 \%$ e especificidade de $93,8 \%$ na diferenciação do mioma submucoso com o intramural na histerossonografia. Estes mesmos autores notaram que as pacientes com mioma submucoso apresentavam uma atrofia endometrial localizada na área de protusão do mioma, mesmo na fase secretora, o que dificultava o diagnóstico do mioma submucoso pela ultra-sonografia transvaginal convencional, especialmente na fase proliferativa precoce. Esta falha foi corrigida com o emprego da histerossonografia $^{16}$. Na presença de miomas submucosos, a HSF tem algumas vantagens e peculiariedades em relação à histeroscopia, pois somente a histerossonografia pode avaliar toda a extensão e profundidade do mioma submucoso e sua vascularização por meio da dopplerfluxometria, ao passo que a histeroscopia só visibiliza uma pequena parte do mioma protuso na cavidade.

A sensibilidade e especificidade da histerossonografia nos casos de hiperplasia endometrial e cavidades normais foram de $100 \%$, superior a maioria dos indices descritos na literatura ${ }^{13,17,18}$. Já Parsons e Lense ${ }^{14}$ obtiveram sensibilidade de 100\% no diagnóstico de hiperplasia endometrial, 
o que está de acordo com os nossos achados.

Os resultados obtidos pelos examinadores I e II foram comparados para cada diagnóstico e mostraram uma correlação estatisticamente significante (IK entre 0,89 e 1,0), provando que a HSG é um método altamente reprodutivel. É importante ressaltar que os exames deste estudo foram gravados em vídeo e analisados dinamicamente, já que somente a análise das fotos dos exames seria insuficiente na definição diagnóstica, por estas serem estáticas. A correlação entre os diagnósticos da histerossonografia e dos métodos "padrão ouro" também foi estatisticamente significante para todas as patologias (IK entre 0,95 e 1,0). Os examinadores não tiveram acesso prévio aos diagnósticos obtidos pelos métodos "padrão ouro", o que elimina a presença de possíveis vícios.

Embora não tenha sido feita a análise da relação custo-benefício da histerossonografia, outros autores investiram nestes estudos chegando à conclusão de que a histeroscopia diagnóstica encarece em média 3,5 vezes, se comparada à histerossonografia ${ }^{10,19}$.

Este estudo foi limitado à realização de histerossonografia em pacientes sintomáticas e com alterações da cavidade uterina diagnosticadas pela ultra-sonografia transvaginal e mostrou ter alta acurácia diagnóstica. A sensibilidade e especificidade da HSF, encontradas neste estudo, deverão ser interpretadas com cautela, para que não se extrapolem os resultados para diferentes grupos de pacientes. Em nenhum momento realizaram-se cálculos de sensibilidade e especificidade da ultra-sonografia transvaginal, por se tratar de uma amostra pré-selecionada e sujeita a vícios de seleção. Da mesma forma estes dados não se prestam para comparação entre a ultra-sonografia transvaginal e HSF. Será importante fazermos, no futuro, um estudo comparativo da histerossonografia com os métodos "padrão ouro", visando definir o valor do método como triagem ("screening") de patologias em uma população assintomática.

Portanto, a partir deste estudo fica claro que a histerossonografia é uma ótima opção dentre os métodos disponiveis para estudo da cavidade uterina, por ser um método altamente reprodutivel entre diferentes examinadores, além de ter alta sensibilidade, especificidade e valores preditivos positivo e negativo no diagnóstico das anormalidades benignas da cavidade uterina, em pacientes com sangramento uterino anormal.

\section{SUMMARY}

Purpose: to determine the role of sonohysterography in the evaluation of abnormalities in the uterine cavity in patients presenting abnormal uterine bleeding, who had previously been selected by transvaginal ultrasonography.

Methods: forty-eight patients presenting abnormal uterine bleeding and changes in the uterine cavity seen by transvaginal ultrasonography were selected, and they were in the menacme or postmenopause period. All patients underwent a sonohysterography, and later a hysteroscopy and/or a hysterectomy. The sonohysterographies were evaluated by two different physicians, and the diagnoses were compared.

Results: the sonohysterography method showed high sensitivity and specificity for the diagnosis of benign pathologies in the uterine cavity. First, in the presence of polyps the sensitivity and specificity rates were 100 and $97 \%$, respectively, second, in the presence of submucous myoma, they were 83 and $100 \%$, and finally, concerning endometrial hyperplasia and normal endometrium, they were $100 \%$. We diagnosed thirty-three cases of polyps, thirteen cases of submucous myoma, four cases of endometrial hyperplasia and three normal cases. The correlation between the diagnoses provided by the two physicians was high.

Conclusions: sonohysterography is a safe and fast method which is very well tolerated by the patient, and has low levels of complications. Its high sensitivity and specificity allow this method to be used for routine diagnosis concerning benign pathologies in the uterine cavity of patients presenting abnormal uterine bleeding.

KEY WORDS: Transvaginal ultrasound. Uterine bleeding. Sonohysterography.

\section{Referências}

1. Halbe HW. Tratado de ginecologia. $1^{\mathrm{a}}$ ed. São Paulo: Roca; 1987. p.854-84.

2. Viscomi F. O impacto da histeroscopia no sangramento uterino anormal da perimenopausa: uma mudança de conceitos. Femina 1996; 24: 843-4.

3. Bailão LA, Bonilla-Musoles F, Machado LE, Rizzi MCS. Ultra-sonografia transvaginal. $1^{\text {a }}$ ed. Ribeirão Preto: Diagnosis; 1991. p.1-7 .

4. Nannini R, Chelo E, Branconi F, Tantini C, Scarselli GF Dynamic echohysteroscopy: a new diagnostic technique in the study of female infertility. Acta Eur Fertil 1981; 12: 165-71.

5. Richman TS, Viscomi GN, DeCherney A, Polan ML, Alcebo LO. Fallopian tubal patency assessed by ultrasound following fluid injection. Radiology 1984; 152:507-10.

6. Randolph JF Jr, Ying YK, Maier DB, Schmidt CL, Riddick DH, Randolph JR Jr. Comparison of real time ultrasonography, hysterosalpingography and laparoscopy/hysteroscopy in the evaluation of uterine abnormalities and tubal patency. Fertil Steril 1986; 46:828-32. 
7. Sackett DL, Haynes RB, Tugwell P. Clinical epidemiology: a basic science for clinical medicine. $1^{\mathrm{a}}$ ed. Boston: Little Brown; 1985. p.1745.

8. Dijkhuizen FP, Brölman HÁ, Potters AE, Bongers MY, Heinz AP The accuracy of transvaginal ultrasonography in the diagnosis of endometrial abnormalities. Obstet Gynecol 1996; 87:345-9.

9. Indman PD. Abnormal uterine bleeding. Accuracy of vaginal probe ultrasound in predicting abnormal hysteroscopy findings. J Reprod Med 1995; 40:545-8.

10.Saidi MH, Sadler RK, Theis VD, Akright BD, Farhart AS, Villanueva GR. Comparison of sonography, sonohysterography, and hysteroscopy for evaluation of abnormal uterine bleeding. $\mathrm{J}$ Ultrasound Med 1997; 16:587-91.

11.Barbero M, Enria R, Pagliano M, et al. Studio comparativo tra isteroscopia diagnostica ed ecografia transvaginale in pazienti com sanguinamento uterino anomalo in epoca peri e postmenopausale. Minerva Ginecol 1997; 49:491-7.

12.Bernard JP, Lecuru F, Darles C, Robin F, De Bièvre $\mathrm{P}$, Taurelle $\mathrm{R}$ Utilisation de l'échographie avec accentuation de contraste comme examen de première intention dans l'exploration de la cavité utérine. J Gynecol Obstet Biol Reprod (Paris) 1997; 27:167-73.
13.Gaucherand P, Piacenza JM, Salle B, Rudigoz RC. Sonohysterography of the uterine cavity: preliminar investigations. J Clin Ultrasound 1995; 23:339-48.

14.Parsons AK, Lense JJ. Sonohysterography for endometrial abnormalities: preliminary results. J Clin Ultrasound 1993; 21:87-95.

15.Cicinelli E, Romano F, Anastasio PS, Blasi N, Parisi C, Galantino P. Transabdominal sonohysterography transvaginal sonography, and hysteroscopy in the evaluation of submucous myomas. Obstet Gynecol $1995 ; 85: 42-7$

16.Fukuda M, Shimizu T, Fukuda K, Yomura W, Shimizu S. Transvaginal hysterosonography for differential diagnosis between submucous and intramural myoma. Gynecol Obstet Invest 1993; 35:236-9.

17.Widrich T, Bradley LD, Mitchinson AR, Collins RL. Comparison of saline infusion sonography with office hysteroscopy for evaluation of the endometrium. Am J Obstet Gynecol 1996; 174:1327-34

18.Rudigoz RC, Salle B, Piacenza JM, de Saint-Hilaire P, Gaucherand P. Étude de la cavité utérine par hystérosonographie. J Gynecol Obstet Biol Reprod (Paris) 1995; 24:697-704.

19.Lindheim SR, Sauer MV. Upper genital-tract screening with histerosonography in patients receiving donated oocytes. Int $\mathrm{J}$ Gynaecol Obstet 1998; 60:47-50.

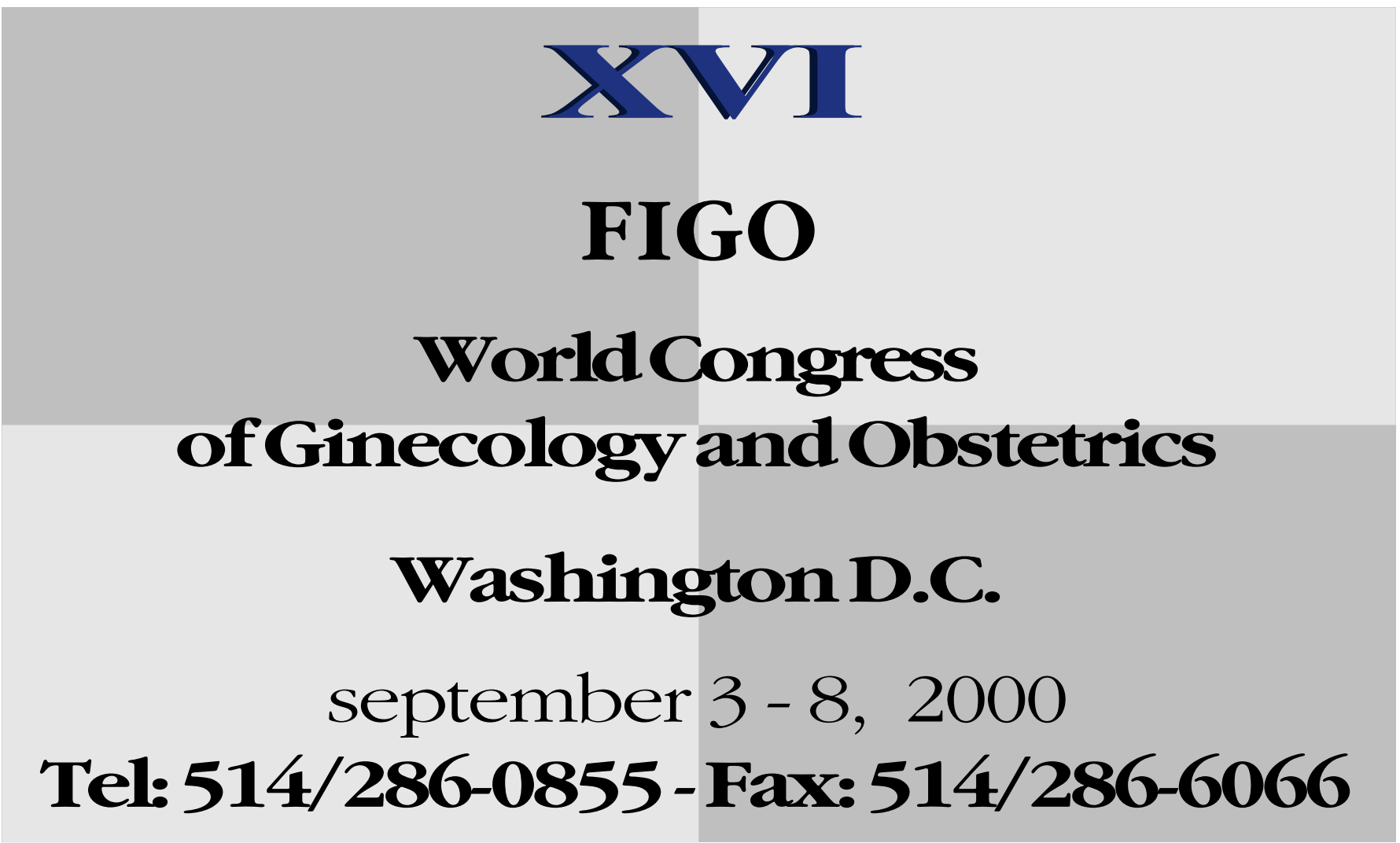

